\title{
Divergent Thermal Conductivity in Three-dimensional Nonlinear Lattices
}

\author{
Hayato Shiba * Satoshi Yukawa ${ }^{*}$ and Nobuyasu Itd \\ Department of Applied Physics, University of Tokyo, \\ Hongo, Bunkyo-ku, Tokyo 113-8656
}

(Dated: July 2, 2018)

\begin{abstract}
Heat conduction in three-dimensional nonlinear lattices is investigated using a particle dynamics simulation. The system is a simple three-dimensional extension of the Fermi-Pasta-Ulam $\beta$ (FPU$\beta$ ) nonlinear lattices, in which the interparticle potential has a biquadratic term together with a harmonic term. The system size is $L \times L \times 2 L$, and the heat is made to flow in the $2 L$ direction with using the Nosé-Hoover method. Although a linear temperature profile is realized, the ratio of energy flux to temperature gradient shows logarithmic divergence with $L$. The autocorrelation function of energy flux $C(t)$ is observed to show power-law decay as $t^{-0.98 \pm 0.25}$, which is slower than the decay in conventional momentum-conserving three-dimensional systems $\left(t^{-3 / 2}\right)$. Similar behavior is also observed in the four-dimensional system.
\end{abstract}

PACS numbers: $63.70 .+\mathrm{h}, 72.25 . \mathrm{Dp}, 44.10 .+\mathrm{i}$

Thermal conduction has been one of the main issues of statistical mechanics for more than a century. Thermal conduction is usually accurately described by the Fourier law

$$
\boldsymbol{J}=-\kappa \nabla T
$$

where $\boldsymbol{J}$ is the heat flux, $\kappa$ is the heat conductivity, and $T$ is the local temperature. The transport coefficient is described by the Green-Kubo formula [1, 2, 3.

$$
\kappa=\frac{k_{B} \beta^{2}}{V} \int_{0}^{\infty} C(t) \mathrm{d} t,
$$

where $C(t)=\langle\boldsymbol{J}(t) \cdot \boldsymbol{J}(0)\rangle$ denotes the equilibrium autocorrelation function of $\boldsymbol{J}(t)$.

In our understanding of the conventional long-time tails of the autocorrelation function, $C(t) \sim t^{-d / 2}$ in a $d$-dimensional system [4, 5, 6], the integral in eq. (2) is expected to diverge in one- and two-dimensional systems, and to converge in three- and higher-dimensional systems. For a finite system with size $L$, the size dependence of the effective transport coefficient $\kappa(L)$, which is defined as the ratio of energy flux to temperature gradient, is obtained by replacing the upper limit of the integral in eq. (2) with the time range $L / v_{s}\left(v_{s}\right.$ denotes a typical phonon velocity). Together with the long-time tail behavior, we obtain $\kappa(L)=a+b L^{1-d / 2}$, where $a$ and $b$ are constants depending on the system, and $L^{1-d / 2}$ should be interpreted to be a logarithmically behaving function $\ln L$ for $d=2$. This argument predicts $\kappa(L) \sim L^{0.5}, \ln L$ and $a+b / L^{0.5}$ for $d=1,2$ and 3, respectively. Therefore, $\kappa(L)$ is expected to diverge for $d=1,2$ and to converge for $d \geq 3$ [7, 8].

Such size dependence has been verified in two- and three-dimensional fluid systems. In the two-dimensional hard-disk system, logarithmic divergence has been confirmed. In the three-dimensional case, $1 / \sqrt{L}$ convergence has been confirmed in the hard-spheres system 7, 9] and in the Lennard-Jones system [10, 11].

In this article, we consider nonlinear lattice systems with total momentum conservation as a model system of insulated solids. In one-dimensional nonlinear lattices 7, 8, 12, 13, 14, 15, 16], the power-law divergence of $\kappa(L)$ has been confirmed, where the estimated value of the exponent is about 0.37 . This value is smaller than the one expected from the above argument. In the two-dimensional case, logarithmic divergence has been observed 17, 18].

The behavior of three-dimensional nonlinear lattice models has not been clarified, however. Only a FermiPasta-Ulam- $\beta$ (FPU- $\beta$ )-like three-dimensional model has been studied, and $1 / \sqrt{L}$ convergence was observed [7]. This model has a natural length in the potential function, and a free boundary condition was used in the direction of heat flow. As a result, the system does not have a crystalline structure in the steady state.

The purpose of this study is to determine whether three-dimensional nonlinear lattices with momentum conserving interaction show $1 / \sqrt{L}$ convergence.

Our model is described by the Hamiltonian

$$
\mathcal{H}=\sum_{i=1}^{N} \frac{\boldsymbol{p}_{i}^{2}}{2}+\sum_{\langle i, j\rangle}\left[\frac{1}{2}\left|\boldsymbol{r}_{i}-\boldsymbol{r}_{j}\right|^{2}+\frac{g}{4}\left|\boldsymbol{r}_{i}-\boldsymbol{r}_{j}\right|^{4}\right],
$$

It is a simple extension of the FPU- $\beta[19]$ chain to higher dimensional lattices. Here, $\boldsymbol{p}_{i}$ and $\boldsymbol{r}_{i}$ denote the momentum and displacement of a particle on lattice point $i$ respectively, and are three-dimensional vectors. All the particles have the unit mass. The summation over $\langle i, j\rangle$ denotes the nearest-neighbor lattice points.

A cubic lattice with a size of $L_{x} \times L_{y} \times L_{z}$ is considered. A periodic boundary condition is used in the $L_{x}$ and $L_{y}$ directions. A fixed boundary condition is used in the $L_{z}$ direction; that is, the particles at both ends 
in the $L_{z}$ direction are coupled to rigid walls through the same interaction potential as that between nearestneighbor particles. Furthermore, the particles at both ends in the $L_{z}$ direction undergo temperature control by the Nosé-Hoover method [20]. The temperature at one end is denoted by $T_{L}$ and the other end by $T_{R}$; therefore, energy flows from the $T_{L}$ end to the $T_{R}$ end along the $L_{z}$ direction after the system reaches a steady state. In summary, the equations of motion are

$$
\begin{aligned}
\dot{\boldsymbol{r}}_{i} & =\boldsymbol{p}_{i} \\
\dot{\boldsymbol{p}}_{i} & = \begin{cases}-\frac{\partial \mathcal{H}}{\partial \boldsymbol{r}_{i}} & \text { (in the bulk) } \\
-\frac{\partial \mathcal{H}^{\prime}}{\partial \boldsymbol{r}_{i}}-\zeta_{i} \boldsymbol{p}_{i} & \text { (at both ends) },\end{cases}
\end{aligned}
$$

where $\zeta_{i}$ denotes the Nosé-Hoover thermostat variables, which obey

$$
\dot{\zeta}_{i}=\frac{1}{Q}\left(\frac{p_{i}^{2}}{3 k_{B} T}-1\right)
$$

$T$ denotes the target temperatures, $T_{L}$ and $T_{R}$, and $Q$ denotes the coupling parameter between $\zeta_{i}$ and $\boldsymbol{p}_{i}$. Boltzmann's constant $k_{B}$ is taken as 1.0. In the following, we fix the parameters as

$$
T_{L}=20.0, T_{R}=10.0, \text { and } Q=1.0 .
$$

In this temperature region, the nonlinear term in the Hamiltonian in eq. (3) has the same order of contribu-

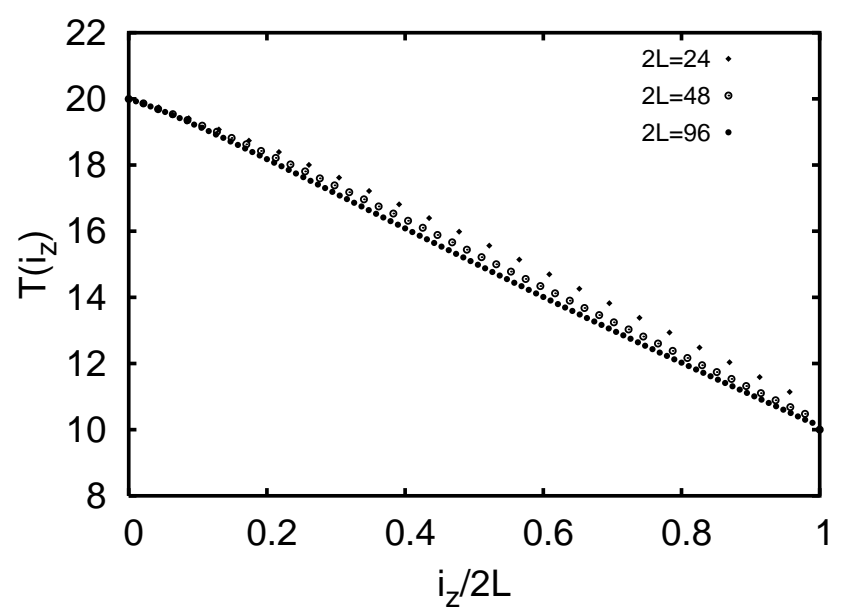

FIG. 1: Temperature profile of three-dimensional FPU- $\beta$ lattice. The size of the system is taken as $L \times L \times 2 L$. Each sequence represents the result for different sizes $2 L=$ 24,48 , and 96 from top to bottom. The horizontal axis shows the $z$-coordinate rescaled by the system size $2 L$, and the vertical axis shows the local temperature averaged over the crosssectional cut in the $x y$-plane. The $3 \sigma$ error is within the marks for each point.

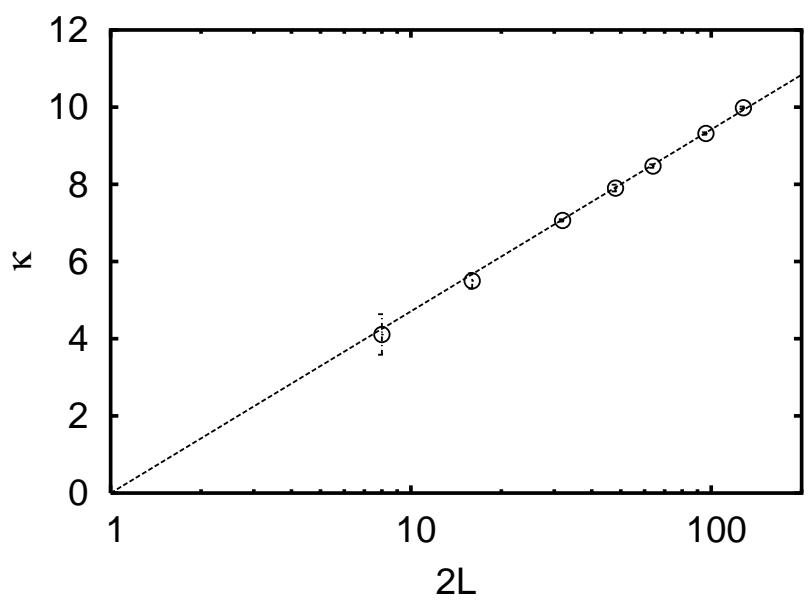

FIG. 2: Size dependence of thermal conductivity in threedimensional FPU- $\beta$ lattices with system size of $L \times L \times 2 L$ given in $\log$ scale. Logarithmic divergence is observed, as shown by the fitted line, $(2.047 \pm 0.004) \times \log 2 L$.

tion as the linear term. Thus, the temperature is sufficiently high for the dynamical evolution to reproduce the thermal state.

First, we study the system of size $L \times L \times 2 L$ to avoid the effects of anisotropy and dimensional crossover. Simulations are carried out using system sizes from $L=4$ to 64 .

Simulations start from a state with all displacements $\boldsymbol{r}_{i}=0$ and with randomly selected momenta $\boldsymbol{p}_{i}$, so that the local kinetic energy profile satisfies a linear temperature profile from $T_{L}$ to $T_{R}$. From this initial state, the system finally reaches a steady state. This initial relaxation process takes about $t=5 \times 10^{4}$ for $L=64$. Afterwards, we sample the local temperature $T\left(i_{z}\right)$, where the particles are labeled in order as $\left(i_{x}, i_{y}, i_{z}\right)$. This is given by the average of the local temperature $T(i)$ of each particle $i$ in the sectional plane of $z=i_{z}$, with $T(i)$ given by the long-time average of the kinetic energy:

$$
T(i)=\left\langle\boldsymbol{p}_{i}^{2} / 3\right\rangle .
$$

The temperature profile is shown in Fig. 1 A typical simulation time is about $1.0 \times 10^{5}$ for $L=64$.h Therefore, the total number of simulation steps per particle for one sample is about $1.0 \times 10^{7}$ which takes slightly more than 1 month using a single core of a $2.2 \mathrm{GHz}$ Opteron processor for the $L=64$ system. Five to eight samples are accumulated for the results. The temperature profile $T(z)$ becomes linear, which shows that thermalization is sufficient in this state.

We estimate the local heat flux at site $i$ with the energy flow using

$$
j_{i}=-\frac{1}{2}\left(\dot{\boldsymbol{r}}_{i+1_{z}}+\dot{\boldsymbol{r}}_{i}\right) \cdot \frac{\partial}{\partial \boldsymbol{r}_{i}} V\left(\boldsymbol{r}_{i+1_{z}}-\boldsymbol{r}_{i}\right),
$$




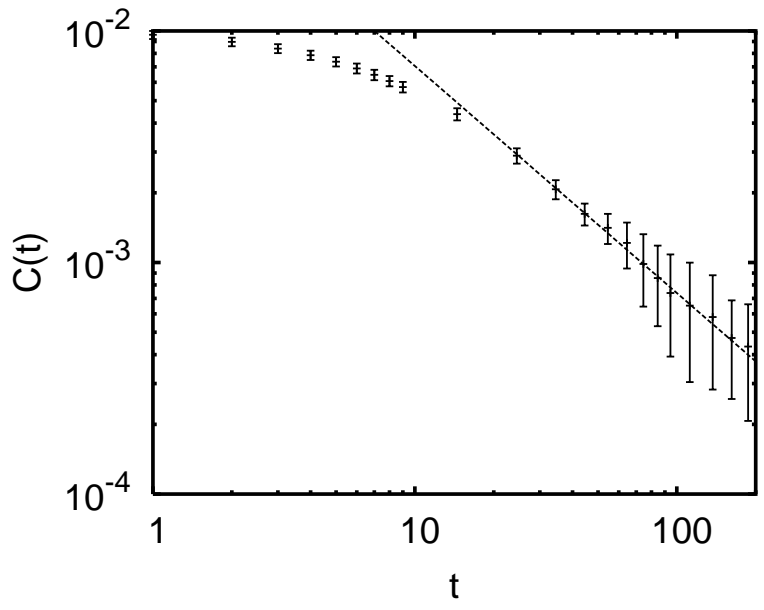

FIG. 3: Autocorrelation function $C(t)=\left\langle J_{z}(t) J_{z}(0)\right\rangle$ in three-dimensional FPU- $\beta$-lattice with system size of $32 \times$ $32 \times 64$. The temperature is set to $T=15$. The periodic boundary condition is applied, and the power-law exponent of the long-time tail behavior is shown by the fitted line $\left(C(t) \sim t^{-0.98 \pm 0.25}\right)$

where $i+1_{z}$ denotes the nearest-neighbor site of the $i$ th particle in the $z$-direction. Heat flux per particle, $J_{z}$, is estimated using

$$
J_{z}=\left\langle\sum_{i} \frac{j_{i}}{N}\right\rangle,
$$

where $\langle\cdot\rangle$ denotes the time average after the simulation reaches to a steady state and $N$ denotes the total number of particles. Then, the thermal conductivity $\kappa(L)$ is estimated using

$$
\kappa(L)=J_{z} /\left(\frac{d T}{d z}\right) .
$$

Figure 2 shows the estimated values of this $\kappa(L)$. A logarithmic divergence is clearly observed for the systems up to $64 \times 64 \times 128$. Divergence of $\kappa(L)$ with $L$ means that the system does not have finite conductivity at the macroscopic limit.

This logarithmic divergence of $\kappa(L)$ is consistent with the long-time behavior of the autocorrelation function, $C(t)=\left\langle J_{z}(t) J_{z}(0)\right\rangle$ in the equilibrium state. Figure 3 shows the estimated values of this $C(t)$ obtained by microcanonical simulation of the same nonlinear lattice without temperature control. The system size is $32 \times 32 \times 64$, and the total energy in the system is adjusted to the internal energy expectation value at temperature $T=15$. A periodic boundary condition is used in all directions in this simulation. In Fig. 3, it is observed that $C(t)$ decays asymptotically as $1 / t^{0.98 \pm 0.25}$ in the longtime limit, which suggests the logarithmic divergence of $\kappa(L)$, as we observed in our nonequilibrium simulation.

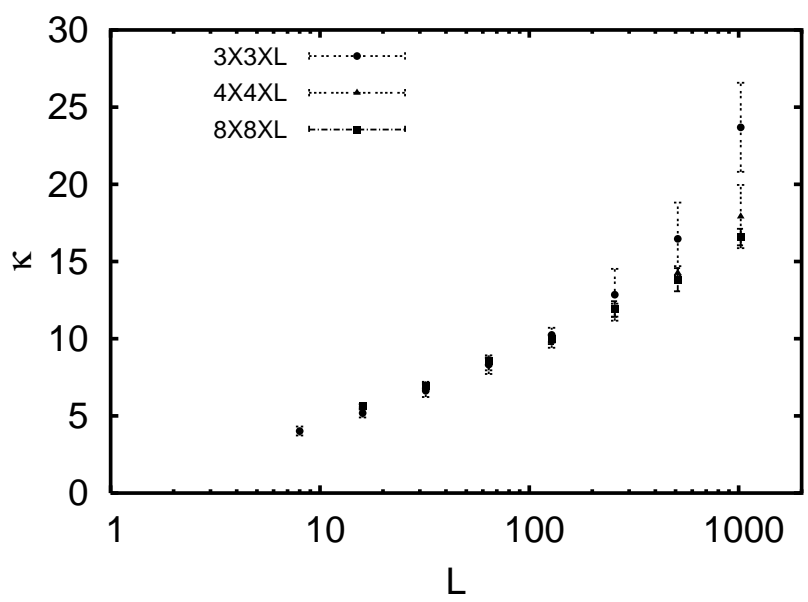

FIG. 4: Size dependence of thermal conductivity in quasi-onedimensional FPU- $\beta$ lattices. Circle, triangle, and square plots respectively represent the thermal conductivities for system sizes of $3 \times 3 \times L, 4 \times 4 \times L$, and $8 \times 8 \times L$.

This is evidence that the divergence of $\kappa(L)$ is simply due to the bulk property of the system, and it is not caused by a boundary effect or by the temperature control.

Secondly, the thermal conductivity of quasi-onedimensional systems is studied. For fixed $L_{x}$ and $L_{y}$, the $L_{z}$ dependence of the thermal conductivity, $\kappa\left(L_{z}\right)$, is estimated. Details of the computer simulation and the model parameters are the same as above. The systems with $L_{x}=L_{y}=3,4$, and 8 are simulated for $L_{z}=8$ to 1024. The results are shown in Fig. [4 Estimated values of thermal conductivity are consistent with the bulk values of the system with the same $L_{z}$ as shown in Fig. 2, for up to $L_{z} \sim 128$, and the longer systems have larger $\kappa$ and show power-law divergence, which was confirmed in various one-dimensional systems. The crossover length from the logarithmic divergence to the power-law increases with thickness $\left(L_{x}=L_{y}\right)$. The length is about 256 or 512 , even for the thinnest $L_{x}=L_{y}=3$ system. Therefore, the crossover length is about 100 times the cross-section size. This result may explain the reason why the convergence of thermal conductivity was confirmed in previous studies that used quasi-one-dimensional systems.

So far, we have observed that conductivity is logarithmically divergent not only in two-dimensional, but also in three-dimensional FPU- $\beta$ lattices. We show here that the conductivity of the four-dimensional FPU- $\beta$ lattice also has the tendency of logarithmic divergence. We prepare the hypercubic lattice with a system size of $L \times L \times L \times 2 L$ in four-dimensional space, and simulate the system using eqs. (3), (4a), and (4b), and boundary conditions similar to those used in the three-dimensional model. The same parameters are used as in the three-dimensional case except for the temperatures, which are set to be $T_{L}=15.0$ 
and $T_{R}=7.5$. The calculated thermal conductivity is shown in Fig. [5 It is notable that the tendency of divergence still appears, as in the three-dimensional case, although we could only calculate up to the system size of $2 L \sim 32$ because larger systems require too much computational load. The result seems to indicate that no dimensionality effect exists in the behavior of the conductivity at the isotropic thermodynamic limit when the dimension is higher than 2 .

The behavior of the model with a natural length and fixed boundary conditions has not yet been investigated, although the convergence of $\kappa(L)$ has been observed in the free-boundary-applied FPU- $\beta$ lattice with a natural length [7]. We simulated such systems with a size of $5 \times$ $5 \times L$. We set the natural length as $l_{0}=100.0$, and the boundary condition in the $x$ and $y$ directions as periodic, and in $z$ direction, the particles at both ends are linked to missing atom which cannot move. The missing atoms on the left and the right sides are separated by a distance of $(L+1) \times l_{0}$. In such a situation, the conductivity $\kappa(L)$ is confirmed to show diverging behavior for a system size of up to $L=512$.

In this study, we investigated heat transport in nonlinear lattices with momentum conservation as a model of insulated solids. The model we used contained no impurities or randomness in mass or interaction, and only the effects of nonlinear interaction and dimensionality contribute to thermalization. We find that such nonlinear lattice systems do not have finite thermal conductivity at the thermodynamic limit, even if they are three- and four-dimensional systems. Contrary to the standard understanding of $t^{-d / 2}$ long-time tail behavior, $t^{-1}$ behavior is widely observed not only in two-dimensional lattices, but also in three- and four-dimensional lattices, and may also appear in higher-dimensional lattices. Therefore, the

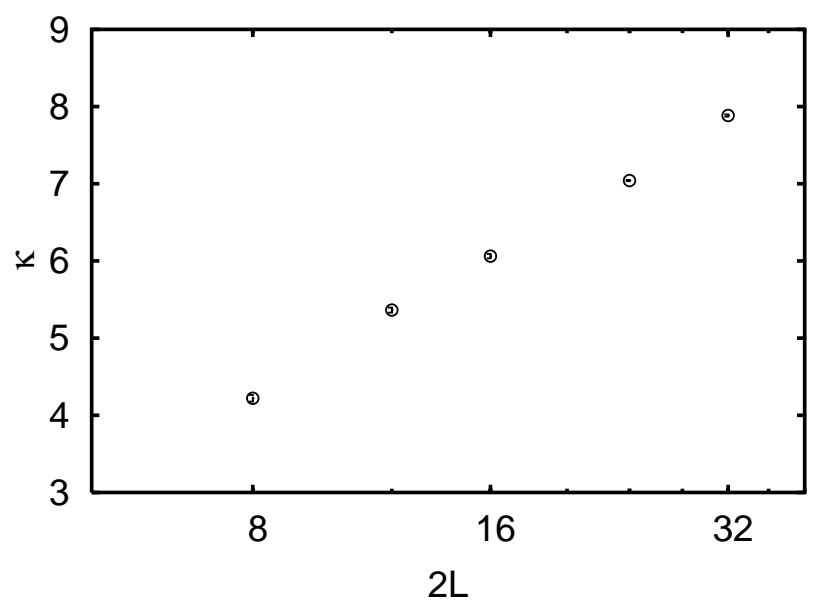

FIG. 5: Size dependence of thermal conductivity in fourdimensional FPU $\beta$ lattices using semilog scale. The size of the system is $L \times L \times L \times 2 L$. standard long-time tail argument cannot be applied in the case of nonlinear lattices. It is natural, because the conventional long-time tail is considered a consequence of viscous modes, which do not exist in our model solids. Our next challenge is to clarify the kinematical origin of the $t^{-1}$ behavior. Of course, studying systems with larger sizes is a topic of future study.

We have not investigated in detail how the conductivity behaves when we increase the values of nonlinear strength $g$ and temperature $T$. However, we confirm a similar divergence of $\kappa(L)$ when $g$ is up to 1.0 and $T$ is up to approximately 100.0. Detailed investigations on the effect of stronger nonlinearity are problems to be investigated.

It is clear that there is still a long way to go before we can model normal heat conduction using nonlinear lattice models. Or, our results may be an indication of a new mechanism of heat flow. The problems concerning the minimum conditions for normal heat conduction and the behavior of heat flow in insulated solids remain unsolved. Heat conduction in three-dimensional models requires further investigation to realize a conclusive model for heat conduction in crystalline insulated solids.

The authors thank the Supercomputer Center, Institute for Solid State Physics, University of Tokyo for the use of the HITACHI SR11000. This work is partially supported by the Japan Society for the Promotion of Science (No. 14080204). The authors gratefully thank Keiji Saito, Takashi Shimada and Koji Azuma for valuable discussions, and Synge Todo for helpful advice.

* Electronic address: shiba@acolyte.t.u-tokyo.ac.jp

$\dagger$ Present address: Department of Earth and Space Science, Graduate School of Science, Osaka University, Osaka 560-0043

‡ Electronic address: ito@ap.t.u-tokyo.ac.jp

[1] R. Kubo: J. Phys. Soc. Jan. 12 (1957) 570.

[2] R. Kubo, M. Yokota and S. Nakajima: J. Phys. Soc. Jpn. 12 (1957) 1203.

[3] M. S. Green: J. Chem. Phys. 22 (1954) 398.

[4] B. J. Alder and T. E. Wainwright: Phys. Rev. A 1 (1970) 18.

[5] Y. Pomeau and P. Résibois: Phys. Rep. 19 (1975) 63.

[6] M. H. Ernst: Physica D 47 (1991) 198.

[7] T. Shimada, T. Murakami, S. Yukawa, K. Saito and N. Ito: J. Phys. Soc. Jpn. 69 (2000) 3150.

[8] S. Lepri, R. Livi and A. Politi: Phys. Rep. 377 (2003) 1.

[9] T. Murakami, T. Shimada, S. Yukawa and N. Ito: J. Phys. Soc. Jpn. 72 (2003) 1049.

[10] F. Ogushi, S. Yukawa and N. Ito: J. Phys. Soc. Jpn. 74 (2005) 827.

[11] F. Ogushi, S. Yukawa and N. Ito: J. Phys. Soc. Jpn. 75 (2006) 073001.

[12] H. Kaburaki and M. Machida: Phys. Lett. A 181 (1993) 85.

[13] S. Lepri, R. Livi and A. Politi: Phys. Rev. Lett. 78 (1997) 
1896.

[14] T. Hatano: Phys. Rev. E 59 (1999) R1.

[15] S. Lepri, R. Livi and A. Politi: Europhys. Lett. 43 (1998) 271

[16] O. Narayan and S. Ramaswamy: Phys. Rev. Lett. 89 (2002) 200601.

[17] A. Lippi and R. Livi: J. Stat. Phys. 100 (2000) 1147.
[18] S. Lepri, R. Livi and A. Politi: Chaos 15 (2005) 015118.

[19] E. Fermi, J. Pasta and S. Ulam: Los Alamos Report No. LA-1940 (1955), later published in E. Fermi, Collected Papers, E. Segré (ed.), University of Chicago Press (1965).

[20] S. Nosé: Prog. Theor. Phys. Suppl. 103 (1991) 1. 